\title{
STRUCTURE OF CLOSE BINARIES. I: POLYTROPES
}

\author{
M. D. T. NAYLOR and S. P. S. ANAND \\ David Dunlap Observatory, University of Toronto, Canada
}

\begin{abstract}
An extension of the method devised by Monaghan and Roxburgh (1965) for rapidly rotating polytropes is used to study the structure of the primary component of a synchronous close binary system. Results are presented for polytropes of index 1.5, 2.0, 3.0, 4.0 and 4.9. We conclude that the extended Monaghan and Roxburgh method can be applied to real stars which are perturbed by both tidal and rotational forces.
\end{abstract}

\section{Introduction}

Recently there has been considerable work by a number of groups on the evolution of stars in binary systems (Plavec, 1968, and references therein). The results have been obtained by using the standard spherically symmetric equations of stellar structure with special boundary conditions (Plavec, 1968), and it was also explicitly assumed that the effects of the centrifugal and tidal distortions are small, and should not seriously change the overall picture of binary star evolution.

It is the purpose of the present investigation to examine this assumption by calculating detailed models which will include both distortion terms. In this our first communication, we will consider the problem of close binary polytropes as they should give an estimate of the accuracy which can be expected from the method we have chosen.

In a famous series of papers Chandrasekhar (1933a, b, c, d) developed a first-order perturbation analysis which he applied to the rotational problem, the tidal problem, and the binary star problem. Unfortunately when the separation between the components is only a few times the undisturbed radius of the primary (i.e. more massive) component, Chandrasekhar's method no longer gives accurate results near the surface of the polytrope unless modified (Monaghan, 1967; Martin, 1970). Since then there has been very limited progress in this direction (Takeda, 1934; Kuiper, 1941; Kopal, 1959; Orlov, 1961).

In the last five years several methods for finding exact solutions to the rotational problem have appeared (James, 1964; Stoeckly, 1965; Ostriker and Mark, 1968). However these methods are cumbersome, require large amounts of computer time, and are difficult to apply to real stars. For these reasons, Monaghan and Roxburgh (1965) (hereinafter referred to as MR) developed a simple perturbation method which gives fairly accurate results for rapid rotation but required much less computer time than the exact treatments. This technique was then used by Roxburgh et al. (1965), Faulkner et al. (1968), Sackmann (1968), and Sackmann and Anand (1970) to determine the structure of rapidly rotating stars.

The MR technique for rotating polytropes can easily be modified to study binary systems. This is accomplished by following the analysis of MR but including the tidal potential as well as the centrifugal potential. Results were obtained for polytropic models of the primary with indices $1.5,2.0,3.0,4.0$, and 4.9. 


\section{Basic Equations and Method of Solution}

Consider two gaseous masses in hydrostatic equilibrium which are revolving in circular orbits about their common centre of mass. Spherical polar coordinates $(r, \theta, \phi)$ are introduced with origin at the centre of mass of the primary and the azimuthal angle $\phi$ is measured from the line joining the centres of mass of the two components. It is assumed that the rotation axis of the primary is perpendicular to the plane of the orbit and $\theta$ is taken to be the colatitude.

The equation of hydrostatic equilibrium for the primary is

$$
\frac{\nabla P}{\varrho}=\nabla V+\nabla V_{\mathrm{T}}+\nabla\left[\frac{1}{3} \omega^{2} r^{2}\left(1-P_{2}(\mu)\right)-\frac{M_{2}}{M_{1}+M_{2}} R \omega^{2} r P_{1}(\lambda)\right],
$$

where $V$ is the gravitational potential of the primary of mass $M_{1}, V_{\mathrm{T}}$ is the tidal potential of the secondary of mass $M_{2}, \omega$ is the uniform angular velocity of the primary about its rotation axis, and synchronism between rotation and revolution is assumed, $R$ is the separation of the mass centres, and $\mu$ and $\lambda$ are equal to $\cos \theta$ and $\sin \theta \cos \phi$ respectively. The term in square brackets is the centrifugal potential. The gravitational and tidal potentials must satisfy Poisson's and Laplace's equations, respectively. It is assumed that the primary can be represented by a polytrope of index $n$, and the following dimensionless variables are introduced,

$$
\begin{aligned}
& \varrho=\varrho_{\mathrm{c}} \sigma^{n}, \quad \alpha=\omega^{2} / 2 \pi G \varrho_{\mathrm{c}}, \\
& r=a x, \quad a^{2}=K \varrho_{\mathrm{c}}^{(1 / n)-1}(n+1) / 4 \pi G,
\end{aligned}
$$

These variables can be substituted into Equation (1) and with the aid of Poisson's and Laplace's equations we obtain

$$
\nabla_{x, \mu, \phi}^{2} \sigma=-\sigma^{n}+\alpha .
$$

The form of the tidal potential to be used in this investigation has been derived by Chandrasekhar (1933b) to order $(\bar{a} / R)^{5}$ where $\bar{a}$ is the mean radius of the secondary,

$$
V_{\mathrm{T}}=\frac{G M_{2}}{R} \sum_{j=1}^{4}\left(\frac{r}{R}\right)^{j} P_{j}(\lambda)+\text { constant }
$$

To obtain the solution we divide the polytrope into two regions. In the inner region $\sigma$ is obtained by employing a first order perturbation analysis, whereas in the outer region it is assumed that the local gravitational potential is determined only by the mass contained in the inner region. The two solutions for $\sigma$ are then matched on a spherical interface.

In the inner region the perturbing forces are small compared to the local gravitational force and following the method outlined by Chandrasekhar (1933a) and MR, $\sigma$ is expanded in a power series and only first order terms are retained, i.e.

$$
\sigma=\theta(x)+\alpha \Psi(x, \mu, \phi) .
$$


Furthermore we develop $\psi$ in terms of a series of surface harmonics,

$$
\Psi=\psi_{0}(x)+\sum_{j=1}^{\infty} \psi_{j}(x) S_{j}(\mu, \phi),
$$

where the $S_{j}(\mu, \phi)$ must satisfy

$$
\frac{\partial}{\partial \mu}\left[\left(1-\mu^{2}\right) \frac{\partial S_{j}}{\partial \mu}\right]+\frac{1}{\left(1-\mu^{2}\right)} \frac{\partial^{2} S_{j}}{\partial \phi^{2}}+j(j+1) S_{j}=0
$$

for each $j$.

In the outer region the technique is somewhat different. First the equation of hydrostatic equilibrium is integrated

$$
\begin{aligned}
& K(n+1) \varrho_{c}^{1 / n} \sigma=V+\frac{1}{3} \omega^{2} a^{2} x^{2}\left[1-P_{2}(\mu)\right] \\
& \quad-\frac{M_{2}}{M_{1}+M_{2}} R \omega^{2} a x P_{1}(\lambda)+\frac{G M_{2}}{R} \sum_{j=1}^{4}\left(\begin{array}{l}
a \\
R
\end{array}\right)^{j} x^{j} P_{j}(\lambda)+\text { constant } .
\end{aligned}
$$

The angular velocity is related to the masses and their separation by Kepler's law

$$
\omega^{2}=G \frac{\left(M_{1}+M_{2}\right)}{R^{3}}(1+\varepsilon),
$$

where the quantity $\varepsilon$ is of order $(\bar{a} / R)^{5}$ (Martin, 1970). Since $\omega^{2}$ appears only in terms of order $(\bar{a} / R)^{2}$, the $\varepsilon$ term will not enter the subsequent analysis which is consistent to order $(\bar{a} / R)^{5}$.

In this region we assume that the density is so low that its contribution to the total mass is negligible and hence the gravitational potential in the outer region is determined by the mass contained in the inner region. This approximation improves with increasing polytropic index. Thus the gravitational potential in the outer region is the Laplace solution.

$$
\Phi=\frac{\beta_{0}}{x}+\frac{\alpha \beta_{1}}{x}+\alpha \sum_{j=1}^{\infty} \frac{S_{j}^{\prime}(\mu, \phi)}{x^{j+1}}
$$

where $\beta_{0}$ and $\beta_{1}$ are constants, the $S_{j}^{\prime}(\mu, \phi)$ satisfy the equation for surface harmonics and $\Phi$ equals $V / K(n+1) \rho_{\mathrm{c}}^{1 / n}$.

The constants and the forms of the surface harmonics are obtained by matching the two solutions on a spherical interface at radius $x=x_{f}$. The method used by MR to obtain the fitting point is to find that value of $x$ for which the errors involved in the inner and outer solutions are of the same order of magnitude. In the final analysis their values of $x_{f}$ were also influenced by the existing tables of the required functions. In any event the results should not be extremely sensitive to the position of the fitting radius, otherwise the method is unworkable. The validity of this assumption will be examined later. For convenience we have chosen the same values of $x_{f}$ as MR.

The continuity conditions show that both $S_{j}(\mu, \phi)$ and $S_{j}^{\prime}(\mu, \phi)$ involve only the 
spherical harmonics $P_{2}(\mu)$ and $P_{j}(\lambda)$ with coefficients $A_{2}$ and $C_{j}$ say, for the $S_{j}(\mu, \phi)$ and similarly $B_{2}$ and $D_{j}$ for the $S_{j}^{\prime}(\mu, \phi)$. Our final solution for the inner region is

$$
\sigma=\theta+\alpha \psi_{0}+\alpha A_{2} \psi_{2} P_{2}(\mu)+\alpha \sum_{j=2}^{4} C_{j} \psi_{j} P_{j}(\lambda)
$$

and for the outer region

$$
\begin{array}{r}
\sigma=\frac{\beta_{0}}{x}+\frac{\alpha \beta_{1}}{x}+\frac{\alpha \beta_{2}}{x^{3}} P_{2}(\mu)+\alpha \sum_{j=2}^{4} \frac{D_{j} P_{j}(\lambda)}{x^{j+1}}+\frac{1}{6} \alpha x^{2}\left[1-P_{2}(\mu)\right] \\
+\frac{1}{2} \frac{M_{2}}{M_{1}+M_{2}} \alpha \sum_{j=2}^{4}\left(\begin{array}{l}
a \\
R
\end{array}\right)^{j-2} x^{j} P_{j}(\lambda)+v_{0}+\alpha v_{1} .
\end{array}
$$

The constants are defined by:

$$
\begin{aligned}
& \beta_{0}=-\theta^{\prime} x^{2}, \quad \beta_{1}=\frac{x^{3}}{3}-x^{2} \psi_{0}^{\prime}, \\
& v_{0}=\theta+\theta^{\prime} x, \quad v_{1}=\psi_{0}+x \psi_{0}^{\prime}-\frac{1}{2} x^{2}, \\
& A_{2}=-\frac{5}{6} \frac{x^{2}}{\left(3 \psi_{2}+x \psi_{2}^{\prime}\right)}, \quad B_{2}=\frac{x^{5}}{6}\left(\frac{x \psi_{2}^{\prime}-2 \psi_{2}}{x \psi_{2}^{\prime}+3 \psi_{2}}\right), \\
& C_{j}=\frac{M_{2}}{M_{1}+M_{2}}\left(\frac{a}{R}\right)^{j-2} c_{j},
\end{aligned}
$$

where

$$
\begin{aligned}
& c_{j}=\frac{1}{2}\left[\frac{(2 j+1) x^{j}}{(j+1) \psi_{j}+x \psi_{j}^{\prime}}\right], \\
& D_{j}=\frac{M_{2}}{M_{1}+M_{2}}\left(\frac{a}{R}\right)^{j-2} d_{j},
\end{aligned}
$$

where

$$
d_{j}=\frac{x^{2 j+1}}{2}\left[\frac{j \psi_{j}-x \psi_{j}^{\prime}}{(j+1) \psi_{j}+x \psi_{j}^{\prime}}\right]
$$

the prime denotes differentiation with respect to $x, j=2,3$, and 4 , and the constants are evaluated at the fitting radius. New integrations were obtained for all functions, and their values, as well as those of the constants, are given in Table I.

For convenience a further simplification is introduced. It was shown by Chandrasekhar (1933c) that the parameters $\alpha, q$, and $\eta$ where $\eta=R / a$ and $q=M_{2} / M_{1}$ are not independent for synchronism but are related by

$$
\alpha=\frac{2(1+q) x_{1}^{2}\left|\theta_{1}^{\prime}\right|}{\eta^{3}}, \quad\left|\theta_{1}^{\prime}\right| \equiv \mid \frac{\mathrm{d} \theta^{\prime}}{\left.\mathrm{d} x_{\mid}\right|_{x=x_{1}}}
$$

which is correct to first-order in $\alpha$; the subscript 1 denotes the value at the Emden radius. 
TABLE I

Function values and constants at the fitting radius

\begin{tabular}{llllll}
\hline$n$ & 1.5 & 2.0 & 3.0 & 4.0 & 4.9 \\
$x_{f}$ & 3.2 & 3.6 & 5.0 & 8.0 & 97.4 \\
$\theta$ & $1.0455(-1)$ & $1.1525(-1)$ & $1.1082(-1)$ & $1.0450(-1)$ & $7.6465(-3)$ \\
$\theta^{\prime}$ & $-2.5875(-1)$ & $-1.8269(-1)$ & $-8.0126(-2)$ & $-2.7957(-2)$ & $-1.8179(-4)$ \\
$\psi_{0}$ & $1.0423(0)$ & $1.2961(0)$ & $2.7202(0)$ & $8.1810(0)$ & $1.5688(3)$ \\
$\psi_{0}{ }^{1}$ & $4.9660(-1)$ & $6.7495(-1)$ & $1.2512(0)$ & $2.3563(0)$ & $3.2434(1)$ \\
$\psi_{2}$ & $4.3134(0)$ & $4.5360(0)$ & $6.5108(0)$ & $1.3843(1)$ & $1.7738(3)$ \\
$\psi_{2}{ }^{1}$ & $1.0255(0)$ & $1.2617(0)$ & $1.9891(0)$ & $3.1887(0)$ & $3.6417(1)$ \\
$\psi_{3}$ & $1.7411(1)$ & $2.2090(1)$ & $4.9772(1)$ & $1.8284(2)$ & $3.0095(5)$ \\
$\psi_{3}{ }^{1}$ & $1.1811(1)$ & $1.4617(1)$ & $2.7318(1)$ & $6.6699(1)$ & $9.2691(3)$ \\
$\psi_{4}$ & $6.3560(1)$ & $9.3676(1)$ & $3.0845(2)$ & $1.8700(3)$ & $3.8377(7)$ \\
$\psi_{4}{ }^{1}$ & $6.7034(1)$ & $9.2461(1)$ & $2.3611(2)$ & $9.2231(2)$ & $1.5760(6)$ \\
$A_{2}$ & $-5.2604(-1)$ & $-5.9504(-1)$ & $-7.0674(-1)$ & $-7.9557(-1)$ & $-8.9145(-1)$ \\
$B_{2}$ & $-1.8427(1)$ & $-2.5151(1)$ & $-5.4356(1)$ & $-1.7732(2)$ & $-8.8761(4)$ \\
$\beta_{0}$ & $2.6496(0)$ & $2.3676(0)$ & $2.0032(0)$ & $1.7889(0)$ & $1.7246(0)$ \\
$\beta_{1}$ & $5.8375(0)$ & $6.8047(0)$ & $1.0384(1)$ & $1.4862(1)$ & $2.6347(2)$ \\
$\nu_{0}$ & $-7.2345(-1)$ & $-5.4242(-1)$ & $-2.8981(-1)$ & $-1.1910(-1)$ & $-1.0060(-2)$ \\
$\nu_{1}$ & $-2.4886(0)$ & $-2.7541(0)$ & $-3.5233(0)$ & $-4.4683(0)$ & $-1.5017(1)$ \\
$c_{2}$ & $1.5781(0)$ & $1.7851(0)$ & $2.1202(0)$ & $2.3867(0)$ & $2.6743(0)$ \\
$c_{3}$ & $1.0675(0)$ & $1.1583(0)$ & $1.3033(0)$ & $1.4167(0)$ & $1.5352(0)$ \\
$c_{4}$ & $8.8644(-1)$ & $9.4333(-1)$ & $1.0329(-1)$ & $1.1018(0)$ & $1.1726(0)$ \\
$d_{2}$ & $5.5282(1)$ & $7.5452(1)$ & $1.6307(2)$ & $5.3197(2)$ & $2.6628(5)$ \\
$d_{3}$ & $2.3084(2)$ & $3.7936(2)$ & $1.4809(3)$ & $1.2384(4)$ & $7.3862(8)$ \\
$d_{4}$ & $1.3130(3)$ & $2.6519(3)$ & $1.9105(4)$ & $4.0729(5)$ & $3.1498(12)$
\end{tabular}

The surface of the polytrope is specified by $\sigma=0$. If $\sigma=0$ and simultaneously $\partial \sigma / \partial x=0$ at $\mu=0, \lambda=1$, then the effective surface gravity at the equator in the direction of the secondary is zero. The result is the so-called critical configuration.

The smallest equipotential surface (in volume) for which the gradient of the total potential is zero on the line joining the centres of mass is known as the inner Lagrangian surface or contact surface. Hence it is obvious that the primary lobe of the contact surface and the critical configuration are identical in our theory.

\section{Discussion of Results}

The parameters $\left(x_{\mathrm{e}}, \eta_{\mathrm{c}}\right)$ appropriate to the critical configuration can be obtained for various $q$ by solving $\sigma=0$ and $\partial \sigma / \partial x=0$ at $\mu=0, \lambda=1$ simultaneously. When $q$ is zero our equations reduce to those of the rotational problem and in Table II the critical values of $x_{\mathrm{e}}$ and $\alpha_{\mathrm{c}}$ are given, and, for comparison, the values of MR and James (1964) are also listed. The values of $\alpha_{c}$ differ markedly for the case $n=1.5$ and the reason for this behaviour can perhaps be understood because the errors involved in the two-zone approximation for this polytropic index are the largest of all five cases. Moreover the difference between the two values obtained by the same method can be explained by noting that the constants $a_{2}$ (of MR) and $B_{2}$ of the present paper are not equal although they should be. It appears that the value of $\psi_{2}^{\prime}\left(x_{f}\right)$ used by MR is incorrect. A similar 
TABLE II

Comparison of the critical models of Monaghan and Roxburgh (MR), James (J) and the present investigation

\begin{tabular}{|c|c|c|c|c|c|}
\hline$n$ & 1.5 & 2.0 & 3.0 & 4.0 & 4.9 \\
\hline$x_{\mathrm{e}}$ & 5.3687 & 6.3748 & 10.1370 & 22.3408 & 257.2197 \\
\hline$x_{\mathrm{e}}(\mathrm{MR})$ & 5.24 & 6.33 & 10.12 & 22.26 & - \\
\hline$x_{\mathrm{e}}(\mathrm{J})$ & 5.3585 & 6.307 & - & - & - \\
\hline$x_{\mathrm{e}} / x_{1}$ & 1.4694 & 1.4645 & 1.4698 & 1.4922 & 1.5004 \\
\hline$\alpha$ & $3.7544(-2)$ & $1.9439(-2)$ & $3.9304(-3)$ & $3.2203(-4)$ & $2.0269(-7)$ \\
\hline$\alpha(\mathbf{M R})$ & $4.10 \quad(-2)$ & $1.99 \quad(-2)$ & $3.95 \quad(-3)$ & $3.27 \quad(-4)$ & - \\
\hline$\alpha(\mathrm{J})$ & $4.3624(-2)$ & $2.1604(-2)$ & $3.932(-3)$ & - & - \\
\hline
\end{tabular}

situation exists for $n=4.0$ but the effect on the critical values of $x_{\mathrm{c}}$ and $\alpha_{\mathrm{c}}$ is small because of the manner in which $B_{2}$ enters the equation.

The accuracy of the results improves with increasing polytropic index since the amount of mass being neglected becomes vanishingly small. A similar behaviour is expected in the binary star problem.

A sample of the binary results is given in Table III for $q=1.0$ and 0.1 where we list

TABLE III

Dimensions of the critical primary for different $n$ and the corresponding Roche surface

\begin{tabular}{|c|c|c|c|c|c|c|}
\hline & & & $q=1$ & & & \\
\hline$n$ & 1.5 & 2.0 & 3.0 & 4.0 & 4.9 & Roche \\
\hline$x_{\mathrm{c}} / \eta_{\mathrm{c}}$ & 0.5333 & 0.5317 & 0.5303 & 0.5296 & 0.5291 & 0.5000 \\
\hline$y_{\mathrm{c}} / \eta_{\mathrm{c}}$ & 0.3832 & 0.3941 & 0.3851 & 0.3851 & 0.3848 & 0.3742 \\
\hline$z_{\mathrm{c}} / \eta_{\mathrm{c}}$ & 0.3601 & 0.3622 & 0.3647 & 0.3650 & 0.3648 & 0.3562 \\
\hline$w_{\mathrm{c}} / \eta_{\mathrm{c}}$ & 0.4258 & 0.4247 & 0.4237 & 0.4231 & 0.4227 & - \\
\hline & & & $q=0$. & & & \\
\hline$n$ & 1.5 & 2.0 & 3.0 & 4.0 & 4.9 & Roche \\
\hline$x_{\mathrm{c}} / \eta_{\mathrm{c}}$ & 0.8305 & 0.8262 & 0.8217 & 0.8193 & 0.8183 & 0.7175 \\
\hline$y_{\mathrm{c}} / \eta_{\mathrm{c}}$ & 0.6550 & 0.6530 & 0.6508 & 0.6493 & 0.6485 & 0.5961 \\
\hline$z_{\mathrm{c}} / \eta_{\mathrm{c}}$ & 0.5598 & 0.5625 & 0.5651 & 0.5648 & 0.5642 & 0.5345 \\
\hline $\boldsymbol{w}_{\mathrm{c}} / \eta_{\mathrm{o}}$ & 0.6917 & 0.6884 & 0.6848 & 0.6828 & 0.6820 & - \\
\hline
\end{tabular}

the principal dimensions of the Roche primary and those obtained in the present work. For $n=4.9$ the largest differences, $\approx 6 \%$ for $q=1.0$ and $\approx 14 \%$ for $q=0.1$ occur in the value of $x_{\mathrm{c}} / \eta_{\mathrm{c}}$ which appears to be quite sensitive to the relative importance of tidal and centrifugal forces, particularly for small $q$. The differences in the other radii are somewhat less. The fraction of these differences resulting from errors intrinsic to the method is difficult to estimate for all cases. However for $q=1.0$ and for all $n$, $x_{\mathrm{c}} / \eta_{\mathrm{c}}$ should equal one-half exactly. It might appear that the difference in $x_{\mathrm{c}} / \eta_{\mathrm{c}}$ between the $n=4.9$ model and the Roche model is a result of our assumption that the secondary can be represented by a mass point (i.e. we neglect terms of order $\left.(\tilde{a} / R)^{6}\right)$, 
in addition to the use of the MR approximation scheme in the primary. However the largest part of this difference arises in the truncation of the expansion of $1 / r^{\prime}$ (Equation (4), Chandrasekhar 1933c) at $j=4$, because it is assumed that $\tilde{a} / R$ is approximately the same size, or larger, than $r / R$. For the surface radii of the critical models this is not necessarily true, especially in the direction of the secondary. Reference to section III.3 of Kopal's monograph (Kopal, 1959) shows that $r / R$ can be approximately 3 times larger than $\bar{a} / R$ for a mass ratio of 0.1 . In the expansion

$$
\begin{aligned}
& 1 \\
& r^{\prime}
\end{aligned}={ }_{R}^{1} \sum_{j=0}^{\infty}\left(\begin{array}{l}
r \\
R
\end{array}\right)^{j} P_{j}(\lambda),
$$

the summation should be continued until $(r / R)^{j}$ is of the order of $(\bar{a} / R)^{6}$. With this correction the dimensions of the $n=4.9$ polytrope and the Roche model agree to 4 significant figures, and in general, for $4.9 \geqslant n>1.5$ the differences in radii between polytropes and the Roche model are less than $1 \%$. A complete second-order theory has been derived by Martin (1970) which reduces these differences by still another factor of 2. However we feel that such accuracy is not necessary in the calculation of stellar models when the errors in the input data (e.g. opacities) can be much larger.

The effect of changes in the fitting radius (e.g. $\pm 10 \%$ of the values previously quoted) has been investigated. In general it seems that an increase of the fitting radius introduces a smaller change in the results than does a decrease of $x_{f}$ and this shows that the results are more sensitive to the mass approximation than to the first-order expansion. In more quantitative terms for the rotating case a $10 \%$ increase in $x_{f}$ results in an increase of $\approx 0.6 \%$ in the critical value of $x_{\mathrm{e}}$ for $n=1.5$ and this decreases to $\approx 0.2 \%$ for $n=4.0$. The changes in $\alpha_{\mathrm{c}}$ are larger and vary from $\approx 4 \%$ for $n=1.5$ to $\approx 1 \%$ for $n=4.0$. When $x_{f}$ is decreased by $10 \%$ the changes are $\approx 3$ times larger than those changes which occur when $x_{f}$ is increased. As expected the changes decrease as $n$ increases. In the binary case similar variations were obtained, with the percentage change in the separation being larger than the change in $x_{\mathrm{e}}$.

In conclusion, we feel that small changes in the fitting radius will result in only minor variations of the critical parameters. It also appears that it is better to overestimate the fitting radius than to underestimate it.

\section{Conclusion}

Since our principal aim is the study of real binary stars rather than polytropes, the underlying purpose of the present investigation has been to determine whether the extended Monaghan-Roxburgh method is capable of treating such a problem. Hence our discussion of the detailed structure of close binary polytropes has deliberately been brief, but a complete and more accurate treatment will appear elsewhere (Martin, 1970).

Encouraged by the results of the present investigation we are extending this method to study the structure of real binary stars. These models will include detailed opacities 
and energy generation rates and where necessary we will allow for radiation pressure, partial electron degeneracy, and convective envelopes. The main sequence systems will be discussed in the second paper of this series.

\section{Acknowledgements}

We are grateful to Mr. P. G. Martin and Mr. G. G. Fahlman for their useful discussions. We are also indebted to the National Research Council of Canada and the Department of University Affairs of the Province of Ontario for their financial support.

\section{References}

Chandrasekhar, S.: 1933a, Monthly Notices Roy. Astron. Soc. 93, 390.

Chandrasekhar, S.: 1933b, Monthly Notices Roy. Astron. Soc. 93, 449.

Chandrasekhar, S.: 1933c, Monthly Notices Roy. Astron. Soc. 93, 462.

Chandrasekhar, S.: 1933d, Monthly Notices Roy. Astron. Soc. 93, 539.

Faulkner, J., Roxburgh, I. W., and Strittmatter, P. A.: 1968, Astrophys. J. 151, 203.

James, R.: 1964, Astrophys. J. 140, 552.

Kopal, Z.: 1959, Close Binary Systems, Chapman and Hall, London.

Kuiper, G. P.: 1941, Astrophys. J. 93, 133.

Martin, P. G.: 1970, submitted to Astrophys. Space Sci.

Monaghan, J. J.: 1967, Z. Astrophys. 67, 222.

Monaghan, J. J. and Roxburgh, I. W.: 1965, Monthly Notices Roy. Astron. Soc. 131, 13.

Orlov, A. A.: 1961, Soviet Astron.-A.J. 4, 845.

Ostriker, J. P. and Mark, J. W.-K.: 1968, Astrophys. J. 151, 1075.

Plavec, M.: 1968, Adv. Astron. Astrophys. Vol. 6, Academic Press, New York, p. 201.

Roxburgh, I. W., Griffith, J. S., and Sweet, P. A.: 1965, Z. Astrophys. 61, 203.

Sackmann, I. J.: 1968, Ph.D. Thesis, University of Toronto.

Sackmann, I. J. and Anand, S. P. S.: 1970, Astrophys. J. (in press).

Stoeckly, R.: 1965, Astrophys. J. 142, 208.

Takeda, S.: 1934, Kyoto Mem., A. 17, 197.

\section{Discussion}

Roxburgh: When we did similar calculations several years ago I do not recollect any difference between the Monaghan and Roxburgh paper and the binary work by Durney and myself. The error for the $n=4.9$ case seems enormous; you seem to be saying that the quadrupole moment of such a centrally condensed polytrope is very large - this I find difficult to accept. Since you can make the error in the approximation technique very small for this case you should be able to calculate the structure to a high degree of accuracy. The external potential can be expanded as a power series at the fitting point and keeping terms up to and including the fourth power should give an accuracy of much better than $1 \%$ since the fitting point is so far inside the polytrope that $r / R \ll 1$ so that $(r / R)^{6} \ll$ 0.01 . All coefficients should be known to a high degree of accuracy, the quadrupole moment should be very small and you should reproduce the Roche model results.

Jackson: I have also calculated some models for close binary members using the same method. They were Cowling models, which include the opacity and nuclear energy generation as simple power laws. My results agree with those presented yesterday by Dr. Thomas. They show that the reduction in luminosity is due almost entirely to rotation and that the increase in volume is due mostly to rotation, with only a small contribution from the tidal force. 